\title{
Transfer of a taste aversion from food to water under various states of deprivation
}

\author{
KATY GILLETTE, W. P. BELLINGHAM, and GERARD M. MARTIN \\ Australian National University, Canberra, A.C.T. 2600, Australia
}

\begin{abstract}
The importance of ingestive contexts (feeding and drinking) and deprivation states to rats' transfer of a taste aversion were examined. In Experiments 1 and 2, rats were trained with novel saccharin-treated foods while either food deprived or food and water deprived. They were then tested with a $1.0 \%$ saccharin solution while either water deprived or food and water deprived. Comparable aversions to the solution were displayed regardless of deprivation states. Two further experiments examined transfer to a .1\% saccharin solution in conjunction with deprivation state change. When both stimulus properties and deprivation were widely discrepant from training to test, reduced transfer was noted. The results suggest that stimulus similarity was a stronger controlling variable than deprivation state similarity in facilitating the transfer of an aversion from a feeding context to a drinking context. The results were viewed as being consistent with the known parameters affecting generalization gradients.
\end{abstract}

It is common knowledge that rats readily learn not to consume novel-flavored substances which predict gastrointestinal distress (Revusky \& Garcia, 1970). Conditioned taste-aversion learning is widely viewed as a unique form of learning, in part due to the ubiquity of the phenomenon. In particular, it can be shown that an animal will transfer an aversion for a particular flavor from the original learning context to a dissimilar context. This transfer effect contrasts with other forms of learning, such as shock avoidance, which appear to be more situation specific (Garcia, Kovner, \& Green, 1970).

Demonstrations of taste aversion transfer have been few. Garcia et al. (1970) demonstrated that rats will avoid flavors which predict illness in locations other than where the flavor was originally presented. Using predatory animals, Gustavson, Kelly, and Sweeney (1976) have shown that an aversion to a lamb-meat patty spiced with lithium chloride transfers to the live prey. Male hamsters, poisoned after exposure to the vaginal secretion of female hamsters, alter their mating responses toward estrous females (Johnston, Zahorik, Immler, \& Zakon, 1978). These studies, although demonstrating the transfer of taste aversions from one context to another, fail to elucidate the variables which may positively or negatively influence transfer effects.

Among the important variables known to affect the transfer of learning phenomena in general are stimulus similarity and response similarity (Ellis, 1965; Mackintosh, 1974, pp. 476-477). The following study sought to evaluate the importance of these variables in a taste aversion paradigm. Transfer pro-

The authors wish to thank Sharon Cornthwaite for technical assistance. cedures were used in order to ascertain the specificity of an aversion with respect to feeding or drinking contexts when training and test cues were similar or dissimilar. If taste is the predominant cue used in the formation of a conditioned aversion, the ingestive context in which the taste occurs should have little effect on the display of an aversion. That is, testing animals in a drinking context after training in a feeding context should not attenuate an aversion. However, a procedure in which rats are food deprived, trained with a novel-flavored food, subsequently water deprived and tested with a similarly flavored solution also incorporates a motivational state change from the training to the test situations. It was anticipated that state changes might interfere with the recall of a conditioned aversion. Peck and Ader (1974) have shown that conditioned aversions are sensitive to state effects when rats trained under conditions of satiety or deprivation are later tested under the opposite condition.

In order to control for, and further investigate, the effect of motivational state, the present study used transfer procedures in which rats were trained and tested in different, similar, or identical states. Rats trained with saccharin-flavored food, under food deprivation, were tested with a saccharin solution while deprived of either water alone or both food and water. Similarly, rats trained with saccharin food while food and water deprived were tested with the saccharin solution while in water-deprived or foodand-water-deprived states.

\section{EXPERIMENT 1}

\section{Method}

The experimental subjects were 64 male Wistar hooded rats, 70-80 days old, bred at the Psychology Department Breeding 
Colony, Australian National University, Canberra. Prior to the initiation of experimental procedures, all rats were group-housed. On Day 1, the animals were moved to individual wire cages $(19 \times 16 \times 16 \mathrm{~cm})$ where they remained for the duration of the experiment. The animals were maintained on Mecon rat cubes and tap water. A light $(0700 \mathrm{~h})$ and dark $(1900 \mathrm{~h})$ cycle was imposed throughout the experiment.

All animals were randomly assigned to four equal groups: food deprived trained, water deprived tested (FD-WD), food deprived trained, food and water deprived tested (FD-FWD), food and water deprived trained, water deprived tested (FWD-WD), and food and water deprived trained and tested (FWD-FWD). In each group of 16 animals, 8 were experimentals and 8 were controls. Food consumption was measured to the nearest $.01 \mathrm{~g}$ and fluid to the nearest $.1 \mathrm{ml}$.

On Day 1, the rats were isolated and the deprivation procedure was begun $(1600 \mathrm{~h})$. The rats were adapted to the deprivation schedule during Days 2-8. Food-deprived rats had access to food for $120 \mathrm{~min}$, beginning at $1600 \mathrm{~h}$. Water was available ad lib except for $30 \mathrm{~min}$ from 1600 to $1630 \mathrm{~h}$. Food- and waterdeprived rats were given food for $120 \mathrm{~min}$, beginning at $1600 \mathrm{~h}$ and water for $10 \mathrm{~min}$ at $1750 \mathrm{~h}$.

The food preparations used for training were saccharin-rolled (SR) food, which served as the CS, and flour-rolled (FLR) food, which served as the control food. Saccharin-rolled food was prepared by moistening food cubes and rolling them in powdered saccharin. In order to expose control animals to similar nongustatory characteristics imparted by SR food, FLR food was similarly prepared using all-purpose flour. On Day 9, pretraining measures of the amount of SR food consumed by control animals and FLR food by experimental animals over 30 min were obtained, during which time water was not available. Water was then returned and normal food was available for the remaining $90 \mathrm{~min}$.

Similarly, on Day 10, the training day, measures of SR food consumption by control animals over 30 min were obtained. Conditioned aversions were produced by intraperitoneal injections of $.3 \mathrm{M}$ lithium chloride ( $\mathrm{LiCl})(12.72 \mathrm{~g}$ of $\mathrm{LiCl}$ per $1,000 \mathrm{ml}$ of distilled water) at a dosage of $1.0 \%$ of the animals' body weights. Injections were administered immediately after the training day 30-min consumption period. Following training, the animals had no access to food or water until $1900 \mathrm{~h}$, when both were provided ad lib.

Days 11-14 were recovery days where food and water were available ad lib. On Day $15(1600 \mathrm{~h})$, water was removed from all FD-WD and FWD-WD animals, while both food and water were removed from FD-FWD and FWD-FWD animals. On Days 16 and 17, WD animals were given water for $10 \mathrm{~min}$ at $1750 \mathrm{~h}$, with food freely available. Animals in FWD groups were given food for $120 \mathrm{~min}$, beginning at $1600 \mathrm{~h}$, and water for $10 \mathrm{~min}$ at $1750 \mathrm{~h}$.

Pretest measures of water consumption were taken on Day 18 to confirm group comparability, and on Day 19 all rats were tested for consumption of $1.0 \%$ saccharin solution $(10 \mathrm{~g}$ of saccharin per $1,000 \mathrm{ml}$ of tap water).

\section{Results}

Three-way analyses of variance were performed on the pretraining, training, pretest, and test day data. The main effects were: experimental vs. control groups, training deprivation conditions (FD or FWD), and testing deprivation conditions (WD or FWD). Figures 1 and 2 summarize the means and standard errors for the four deprivation conditions.

The only significant effect on the pretraining and training days was experimental vs. control $[\mathrm{F}(1,56)$ $=134.3, \mathrm{p}<.001$, and $\mathrm{F}(1,56)=156.1, \mathrm{p}<.001$, respectively]. These differences are due to reduced

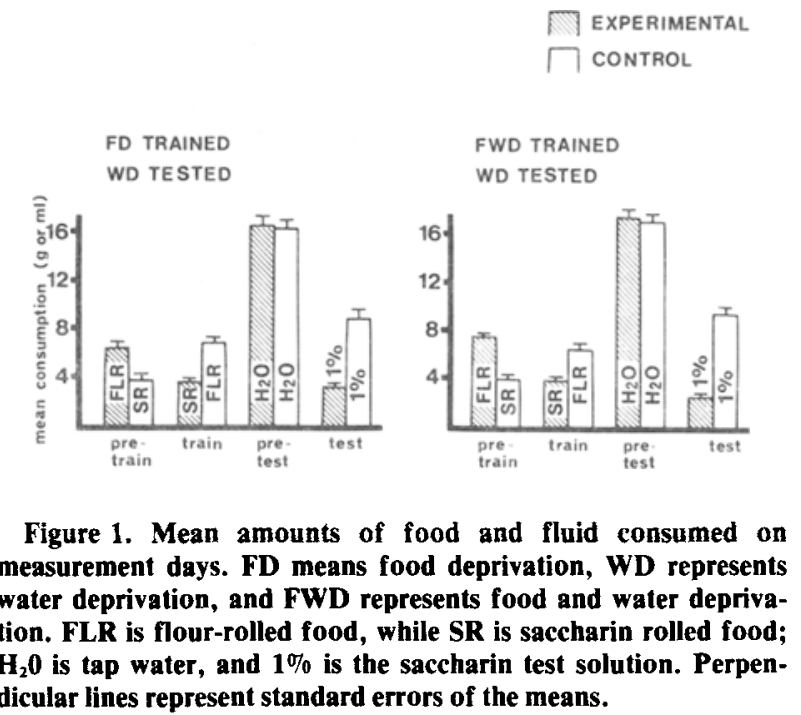

dicular lines represent standard errors of the means.
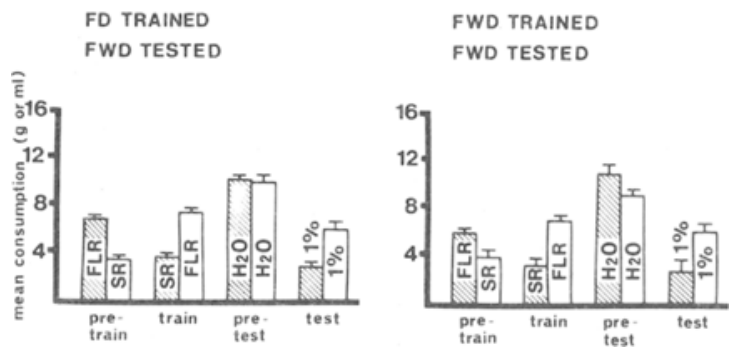

Figure 2. See caption for Figure 1.

SR food consumption by control animals on the pretraining day and by experimental animals on the training day. Analysis for pretest day indicated that WD tested groups drank significantly more water than FWD tested groups $[F(1,56)=252.7, p<$ $.001]$. No other differences on pretest approached significance. On the test day, an aversion for $1.0 \%$ saccharin solution was demonstrated by the experimental groups $[F(1,56)=170.5, p<.001]$. A significant test-deprivation main effect $[F(1,56)=29.4$, $\mathrm{p}<.001]$ and an Experimental/Control Group by Test Deprivation interaction $[\mathrm{F}(1,56=27.1, \mathrm{p}<.001]$ indicated that increased test day consumption for the WD tested groups was entirely attributable to the control group.

\section{Discussion}

In summary, the results indicate transfer of taste aversions regardless of changes in deprivation state from training to testing conditions. That is, the transfer was essentially equivalent (see Figures 1 and 2) from the most similar (FWD-FWD) to the most 
different (FD-WD) training to test conditions. It is recognized that water-deprived animals are to some degree food deprived (Bolles, 1961; Kutscher, 1969; Verplanck \& Hayes, 1953). The reverse is apparently not as certain. Although food-deprived rats reduce water intake (Adolph, 1947; Bolles, 1961; Cizek \& Nocenti, 1965; Kutscher, 1969), the reduction is solely due to that drinking associated with food intake and does not affect basal requirements (Cizek \& Nocenti, 1965). Consequently, one would expect that the FD-WD group would provide the best example of whether a training to test change in deprivation would interfere with the transfer of an aversion.

Pilot data on deprivation state showed that during 10 days of deprivation FD animals consumed the same amount of food as WD animals over each 24-h measurement but drank significantly more water than WD rats under the present deprivation procedures. The animals in the FD group ate an average of $11.4 \mathrm{~g}$ of food during each daily 120 -min feeding session and drank an average of $29.5 \mathrm{ml}$ of ad-lib tap water daily. The animals in the WD condition, however, ate an average of $12.3 \mathrm{~g}$ of ad-lib food daily and drank an average of $12.9 \mathrm{ml}$ of tap water during each 10-min drinking session. After $48 \mathrm{~h}$ recovery on ad-lib food and water, FD and WD groups' food consumption averaged 27.7 and $28.2 \mathrm{~g}$, respectively, while their water consumption averaged 37 and $39.4 \mathrm{ml}$, respectively. Consequently, going from food deprivation during training to water deprivation during testing can be classified as an alteration in internal state. As indicated, no evidence for any difference or trend in performance due to state changes was obtained.

Similarly, changes in extragustatory cues such as form (e.g., brightness, shape, wet vs. dry), location (i.e., floor of cage vs. tube on side of cage), or response topography (i.e., eating vs. drinking) did not prevent transfer. How much importance that should be placed on these extragustatory cues must be assessed in light of probable overshadowing of familiar environmental and response-generated cues by the novel and salient saccharin taste. This is not to say that such context cues could not enter into an independent association with an illness UCS. In the present experiment, it seems reasonable to assume that context cues were relatively less salient than the taste cue due to the fact that the majority of ingestive context cues were highly familiar. Although the brightness and texture of SR food was relatively novel, it must be remembered that FLR food shares these characteristics and had been presented $24 \mathrm{~h}$ prior to training in the absence of any UCS occurrence. It cannot be categorically stated that the taste cue acquired all available associative strength at the expense of the context cues with which it was associated, simply because a $1.0 \%$ saccharin fluid vs. fluid control was not included as a no-transfer comparison. However, it is evident that nontaste cues did not interfere with the transfer of a taste aversion, since attenuation was not apparent. It is probably the case that positive transfer was largely due to the familiarity and consequent overshadowing of contextual cues.

It is clear that the only significant difference in the pattern of results among the most similar to the most different deprivation transfer conditions are attributable to higher levels of fluid consumption in the WD groups during testing. It is not known why the WD tested animals were seemingly more thirsty than FWD tested animals but the difference, on the test day, was only manifested by the control subjects, there being no significant differences across test conditions for experimental groups.

Although there were significant reductions in the amount of SR food consumed by both experimental and control animals, indicating neophobia and/or unpalatability, the transfer of an aversion could not be attributed to induced or enhanced neophobia. Both experimental and control subjects received $\mathrm{LiCl}$ injections on training day while exposure to the novel SR food was equivalent. Neither can sensitization account for the results due, again, to the fact that all groups received $\mathrm{LiCl}$ injections.

\section{EXPERIMENT 2}

A criticism of Experiment 1 is that transfer of a saccharin food aversion to saccharin solution could be an artifact due to incorporation of the saccharin powder from mouth, paws, or fur to the water present after training. In spite of attempts to minimize the probability that the saccharin taste could be associated with drinking as well as feeding, there is a possibility that the transfer observed was a result of this procedural artifact.

In the following experiment, ordinary food cubes were dipped in a strong saccharin solution in order to further reduce the possibility of artifactual transfer. In all other respects, this experiment was a replication of the first.

\section{Method}

Sixty-four male Wistar hooded rats were trained and tested under the conditions described in the Method section for Experiment 1 . Saccharin-dipped (SD) food was prepared by dipping food cubes, for approximately $5-10 \mathrm{sec}$, in a $20.0 \%$ saccharin solution ( $200 \mathrm{~g}$ of saccharin per $1,000 \mathrm{ml}$ of tap water), while plaindipped (PD) food consisted of cubes similarly dipped in tap water, and dried for $24 \mathrm{~h}$. All other aspects of the procedure were identical to those of Experiment 1.

\section{Results and Discussion}

Three-way analyses of variance were performed as in Experiment 1. The results were similar to those found with SR food with one notable exception. The pretraining $[F(1,56)=41.3, p<.001]$ and 
training day $[\mathrm{F}(1,56)=19.4, \mathrm{p}<.001]$ consumption level of SD food was significantly higher than that of PD food. These results indicate a preference for SD food and no neophobia surviving the 30-min feeding period. There was also significantly increased food consumption by the FWD groups over the FD groups on the training day $[F(1,56)=94.9, p<.001]$.

As before, the pretest water consumption by WD tested groups was significantly higher than by FWD tested groups $[F(1,56)=181.9, p<.001]$. On the test day, all experimental groups consumed less saccharin solution than control groups $[\mathrm{F}(1,56)=94.9, \mathrm{p}<$ .001 ]. There was marginally higher consumption by WD tested groups $[F(1,56)=4.7, p<.05]$, attributable to WD control subjects.

The results are essentially a replication of Experiment 1 , indicating transfer across ingestive context cues and deprivation changes. The fact that SD food was preferred provides further evidence that transfer cannot be attributed to enhanced neophobia. Again, there was no indication of sensitization being a significant factor in producing the obtained results.

\section{EXPERIMENT 3}

In the preceding experiments, a highly concentrated test solution (1.0\% saccharin) was chosen in order to maximize taste-cue similarity from training to test. The following experiment used a weaker (.1\% saccharin) test solution in order to determine whether failure to transfer could be demonstrated as a function of increased taste cue discrepancy.

\section{Method}

Sixteen rats (eight experimental, eight control) were trained with SR or FLR food under the FD condition and tested WD. The deprivation procedures and food preparations were identical to the relevant condition of Experiment 1 (i.e., the FD-WD condition), except that the test solution was $.1 \%$ saccharin $(1 \mathrm{~g}$ saccharin per $1,000 \mathrm{ml}$ tap water). Following the first test with the saccharin solution, all animals were food deprived and allowed water ad lib. Twenty-four hours later, the animals were presented with SR food for $30 \mathrm{~min}$.

Another 16 animals ( 8 control, 8 experimental) were trained and tested on $.1 \%$ saccharin in which the training and testing deprivation condition was WD-WD. The purpose of these groups was to assess the magnitude of an aversion conditioned directly to $.1 \%$ saccharin in the appropriate water-deprived state at the time of training and testing. The deprivation and training procedures were identical to the water-deprivation condition for the FWDFWD group in Experiment 1 except that the training and test solution was $.1 \%$ saccharin.

\section{Results and Discussion}

Separate one-way analyses of variance were performed on all measurement days (see Figure 3). The results of the FD-WD transfer group indicated significantly reduced consumption of SR food by the controls on the pretraining day $[F(1,14)=195.7$, $\mathrm{p}<.001]$ and by the experimental group on the

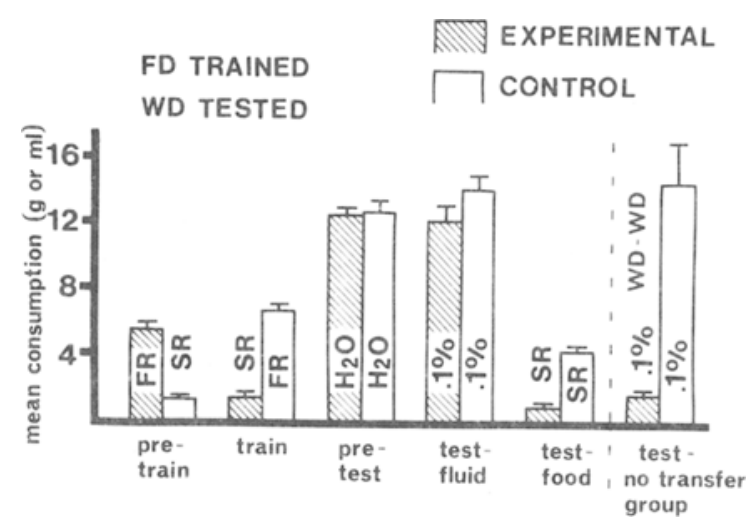

Figure 3. Mean amounts of food and fluid consumed on measurement days. FD is food deprivation and WD water deprivation. FR stands for flour-rolled food and SR for saccharinrolled food. Tap water $\left(\mathrm{H}_{2} \mathrm{O}\right)$ and $.1 \%$ saccharin are the fluids measured. To the right of the dashed perpendicular line is test day consumption of rats trained and tested WD to a $.1 \%$ saccharin solution. Perpendicular lines represent standard errors of the means.

training day $[\mathrm{F}(1,14)=184.7, \mathrm{p}<.001]$. There were no significant differences in tap water consumption on the pretest day $[\mathrm{F}(1,14)=.1, \mathrm{p}>.75]$ or in saccharin consumption on the first test day $[F(1,14)$ $=2.7, \mathrm{p}>.25]$. However, a significant aversion for SR food was shown on the second test day $[F(1,14)$ $=167.2, \mathrm{p}<.001]$. Test day consumption of $.1 \%$ saccharin solution by experimental animals was significantly lower than by control animals in the notransfer (WD-WD) group $[\mathrm{F}(1,14)=101.2, \mathrm{p}<.001]$.

The conditions for the transfer group in this experiment were essentially those of the FD-WD groups in Experiments 1 and 2 except for a change in the concentration of the saccharin solute from $1.0 \%$ to $.1 \%$. The failure to demonstrate transfer, then, is likely to be largely due to generalization decrement along the taste dimension.

\section{EXPERIMENT 4}

Although the failure to transfer a SR food aversion to $.1 \%$ saccharin solution has been attributed to stimulus generalization decrement, it is possible that changed deprivation states amplify the decrement when sufficiently disparate taste cues are used for training and testing. The relative magnitudes of the transferred aversions found in the first two experiments cannot be assessed since a saccharin-solutiontrained/saccharin-solution-tested group was not included.

The following experiment was conducted to assess the effect of changed deprivation states under the condition of taste-cue disparity. The animals were trained and tested as in Experiment 3, with the exception that training and testing occurred under 
similar (FWD-WD) and identical (FWD-FWD) rather than different deprivational conditions. If changed state played any role in the prevention of a transferied taste aversion, the animals in the following experiment would be expected to show some transfer, since the training state is partially or entirely reinstated on the test trial.

\section{Method}

Six experimental and six control animals were trained with SR food and tested with $.1 \%$ saccharin solution. This procedure differs from that of the FWD-WD group in Experiment 1 only with respect to the concentration of the test solution, and from Experiment 3 only with respect to using similar rather than different deprivation states during training and testing. The inclusion of the relevant testing deprivation condition at the time of training was an attempt to minimize change of deprivation state while ensuring reasonable motivation for food consumption during SR food training. Another six experimental and six control animals were trained as above, with the exception that deprivation states were identical at the time of training and testing, i.e., FWD-FWD.

\section{Results and Discussion}

A two-way analysis of variance on test day consumption showed that experimental animals drank significantly less than controls on the test day $[F(1,20)=64.8, p<.001]$, that there was a difference between the two deprivation conditions $[F(1,20)$ $=30.9, \mathrm{p}<.001]$, and that there was no significant interaction $(p>1)$. Scheffé multiple comparisons showed that: (1) FWD.WD experimental animals drank reliably less than FWD-WD controls $(\mathrm{t}=-4.7, \mathrm{p}<.05)$, (2) FWD-FWD experimental animals also drank reliably less than FWD-FWD controls $(t=-6.7, p<.05)$, (3) FWD-WD experimental animals drank more than FWD-FWD experimentals ( $\mathrm{t}=4.9, \mathrm{p}<.05)$, and (4) FWD-WD controls and FWD-FWD controls did not differ $(\mathrm{t}=$ $2.9, \mathrm{p}>.05)$. In brief, although both experimental groups displayed a significant transfer of an aversion, identical deprivation state conditions (FWDFWD) facilitated a greater transfer than did similar conditions (FWD-WD).

Figure 4 indicates that, although the aversions obtained were significant, they were not as great as that demonstrated by the no-transfer group in Experiment 3. Since control group consumption was similar in Experiments 3 and 4, and since variability was relatively low, an estimate of percentage transfer can be obtained using the formula $(C-E / C) \times$ 100 , where $E$ refers to the experimental group mean and $C$ the control group mean of test day consumption. For the $.1 \%$ trained and tested WD-WD group (see Figure 3, "no transfer" group), percentage transfer was $88.2 \%$ (i.e., experimentals drank $88.2 \%$ less than the amount drunk by controls). In other words, the maximum expected aversion for $.1 \%$ saccharin which could be demonstrated in any of the food-to-fluid transfer conditions in Experiments 3 and 4 would be an $88.2 \%$ drop in consumption by

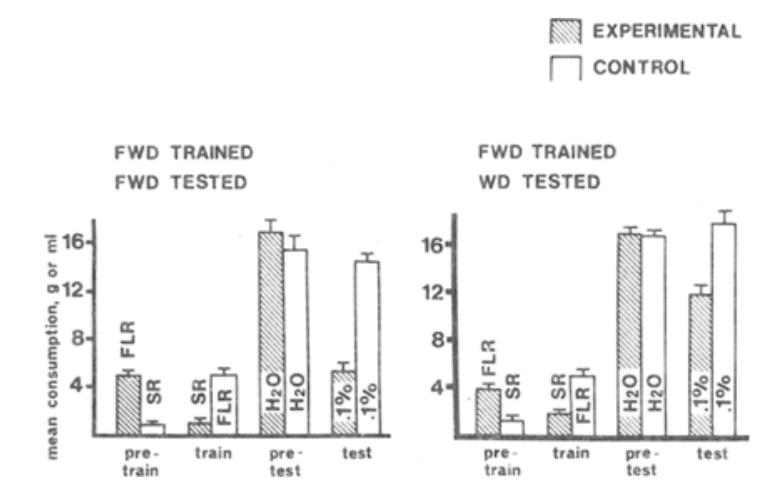

Figure 4. Abbreviations are as for Figure 1, except that the test fluid was . $1 \%$ saccharin.

experimental animals vs. controls. In the present experiment, under FWD-FWD deprivation conditions, transfer of a SR food aversion to $.1 \%$ saccharin was $62.0 \%$. The discrepancy between the WD-WD and FWD-FWD of $26.2 \%$ indicates attenuation due to transfer variables, e.g., food vs. fluid. Under the condition of similar deprivation states, FWD-WD, in the present experiment transfer was $34.2 \%$, indicating a decrease in the strength of the aversion attributable to alterations in deprivation state. For the FD-WD deprivation group of Experiment 3, transfer was $14.7 \%$, showing little or no transfer. These results can be interpreted as indicating that transfer across discrepant taste cues increased with increasing similarity of deprivation state between training and testing.

The internal cues correlated with motivational states appear to become more predominant with increasingly ambiguous circumstances. That is, when both taste stimulus and ingestive context discrepancies are great, stable state cues facilitate transfer of an aversion.

\section{GENERAL DISCUSSION}

The results of the above experiments indicate that rats are capable of transferring a conditioned aversion from a novel flavored food to a similarly flavored solution (Experiments 1 and 2). When the training and test cues are more disparate, transfer is impeded (Experiment 3). Providing the animal with relatively more constant motivational state cues, when taste cues were dissimilar, facilitated transfer (Experiment 4). These results are consistent with the notions that conditioned aversions are displayed independently of a particular context (Garcia et al., 1970) and that transfer of learning is dependent on stimulus similarity and, to some extent, on internal state cue similarity (Mackintosh, 1974, pp. 476-477). The results are also consistent with findings showing that particular ingestive acts are not necessarily a factor in the manifestation of a taste aversion (Bradley \& Mistretta, 1971; Brown, Stewart, \& Hall, 
1976; Domjan \& Wilson, 1972). Somewhat surprising was the relative lack of changes in deprivation state influencing the degree of transfer, given Peck and Ader's (1974) results. In their study, rats trained with a saccharin solution while under water deprivation showed no aversion for the same saccharin solution when satiated, and vice versa. The possibility exists that the states of deprivation and satiety were sufficiently different to produce retrieval failures, while the deprivational procedures used in the present study may not have produced such markedly different motivational states. Another difference is that the two-bottle test procedure used by Peck and Ader may be more sensitive to state changes than the singlebottle technique used in the present study.

The findings that neither altered ingestive contexts nor motivational state changes necessarily interfere with a taste-illness association attest to the wide generalizability of conditioned taste aversions. There are at least two possible reasons for this. First, few potentially available cues other than taste acquire noticeable associative strength in a single conditioning trial and, therefore, variations in these other familiar cues have little effect in impeding the display of an aversion. The possibility that such an event occurred in these experiments was discussed in Experiment 1.

A second consideration is that the conditioned aversion paradigm is well suited, in terms of known parameters influencing generalization, for such widespread generalization. It is well known that punishment procedures produce increased generalization (e.g., Logan, 1976, p. 149) and that generalization is maximal early in training. Under ideal conditions of relatively few training trials and a punishing reinforcer, intermodal generalization has been demonstrated (Thompson, 1959). One-trial lithium chloride induced aversions would qualify on both these counts.

\section{REFERENCES}

Adolph, E. F. Urges to eat and drink in rats. American Journal of Physiology, 1947, 151, 110-125.
Bolles, R. C. The interaction of hunger and thirst in the rat. Journal of Comparative and Physiological Psychology, 1961 , 54, 580-584.

Bradley, R. M., \& Mistretta, C. M. Intravascular taste in rats as demonstrated by conditioned aversion to sodium saccharin. Journal of Comparative and Physiological Psychology, 1971, $75,186-189$.

Brown, R. T., Stewart, R. I., \& Hall, T. L. Extinction of a taste aversion in the absence of the consummatory response. Animal Learning \& Behavior, 1976, 4, 213-216.

Cize K, L. J., \& Nocenti, M. R. Relationship between water and food ingestion in the rat. American Journal of Physiology, $1965,208,615-620$.

Domjan, M., \& Wilson, N. E. Contribution of ingestive behaviors to taste-aversion learning in the rat. Journal of Comparative and Physiological Psychology, 1972, 80, 403-412.

ELLIS, H. The transfer of learning. New York: Macmillan, 1965.

Garcia, J., Kovner, R., \& Green, K. F. Cue properties or palatability of flavors in avoidance learning. Psychonomic Science, 1970, 20, 313-314.

Gustavson, C. R., Kelly, D. J., \& Sweeney, M. Prey-lithium aversions I: Coyotes and wolves. Behavioral Biology, 1976, 17, 61-72.

Johnston, R. E., Zahorik, D. M., Immler, K., \& Zakon, H. Alterations of male sexual behavior by learned aversions to hamster vaginal secretion. Journal of Comparative and Physiological Psychology, 1978, 92, 85-93.

KUTSCHER, C. L. Species differences in the interaction of feeding and drinking. Annals of the New York Academy of Sciences, 1969,154, 539-551.

Mackintosh, N. J. The psychology of animal learning. New York: Academic Press, 1974.

Log AN, F. A. Fundamentals of learning and motivation. Dubuque, Iowa: Brown, 1976.

Peck, J. H., \& AdER, R. Illness-induced taste aversion under states of deprivation and satiation. Animal Learning \& Behavior, 1974, 2, 6-8.

Revusky, S. H., \& Garcia, J. Learned associations over long delays. In G. H. Bower \& J. T. Spence (Eds.), The psychology of learning and motivation: Advances in research and theory (Vol. 4). New York: Academic Press, 1970.

Thompson, R. F. Effect of acquisition level upon the magnitude of stimulus generalization across sensory modality. Journal of Comparative and Physiological Psychology, 1959, 52, 183-185.

VERPLANCK, W. S., \& HAYES, J. R. Eating and drinking as a function of maintenance schedule. Journal of Comparative and Physiological Psychology, 1953, 46, 327-333.
(Received for publication February 23, 1978; revision accepted December $7,1978$. ) 